Archives

\title{
Les origines du discours civique mexicain
}

\section{Carlos Herrejon Peredo}

\section{(apenEdition Journals}

\section{Édition électronique}

URL : http://journals.openedition.org/ccrh/2692

DOI : $10.4000 /$ ccrh.2692

ISSN : $1760-7906$

Éditeur

Centre de recherches historiques - EHESS

Édition imprimée

Date de publication : 14 octobre 1995

ISSN : 0990-9141

\section{Référence électronique}

Carlos Herrejon Peredo, «Les origines du discours civique mexicain», Les Cahiers du Centre de Recherches Historiques [En ligne], 14-15 | 1995, mis en ligne le 27 février 2009, consulté le 01 mai 2019. URL : http://journals.openedition.org/ccrh/2692 ; DOI : 10.4000/ccrh.2692

Ce document a été généré automatiquement le 1 mai 2019.

Article L.111-1 du Code de la propriété intellectuelle. 


\title{
Les origines du discours civique mexicain
}

\author{
Carlos Herrejon Peredo
}

1 Le discours civique dont je vais parler est un discours rhétorique. La rhétorique est l'art de la persuasion; elle est également l'art de bien dire, c'est à dire d'enseigner, de convaincre et de plaire à l'aide de la parole. On connaît le malentendu qui frappa la rhétorique, réduite à un vieil oripeau et par conséquent abandonnée. Mais, ces dernières années, sa véritable reconnaissance dans diverses études a conduit à son réexamen. Dans le champ de la philosophie, une oeuvre pionnière a été le Traité de l'Argumentation de Perelman et Olbrechts-Tytecal ${ }^{1}$. Dans les sciences du langage, des manuels de rhétorique (comme celui de Lausberg²) paraissent à nouveau. D'autres études mettent la rhétorique en relation avec les sciences humaines ou sociales, comme le recueil de Roberts et Good ${ }^{3}$ Indépendamment de ce réexamen, l'histoire s'est intéressée aux expressions rhétoriques en vigueur à un moment donné. Celles-ci appartenaient à une culture, aux travaux et aux jours des hommes qui ont laissé cette trace. Des historiens se sont ainsi montrés attentifs aux contenus doctrinaux du discours, comme Joël Saugnieux dans son livre sur les jansénistes et le renouveau de la prédication en Espagne dans la seconde moitié du XVIII ${ }^{e}$ siècle ${ }^{4}$. D'autres ont fait une histoire de la rhétorique dans une perspective large : Marc Fumaroli dans son ouvrage exemplaire, L'âge de l'éloquence ${ }^{5}$. Dans le cas du Mexique, Ernesto de la Torre a publié un recueil de discours civiques septembristes et Enrique Plasencia, dans Independencia y nacionalismo a la luz del discurso conmemorativo (1825-1867), utilise les discours comme source pour confirmer ou compléter les épisodes de l'histoire nationale auxquels ils correspondent ${ }^{6}$. Pour ma part, je voudrais considérer l'évolution du discours civique comme une tradition, à la fois littéraire et politique, aux racines profondes.

2 Le discours civique a eu une grande importance dans le Mexique du XIX ${ }^{\mathrm{e}}$ siècle. On en a une preuve dans le grand nombre d'écrits de ce genre inventoriés dans le Catàlogo de la Coleccion Lafragua de Lucina Moreno ou dans les fonds de Condumex ${ }^{7}$. Il s'agit d'un discours rhétorique prononcé principalement au cours du mois de septembre, à l'occasion de la commémoration de l'Indépendance nationale. Année après année, voyaient 
publiquement le jour plusieurs des nombreux discours septembristes prononcés à Mexico et dans des villes de province.

3 En règle générale, le discours civique constituait une partie d'une célébration qui comprenait d'autres manifestations comme le défilé, dans les rues préalablement pavoisées, des autorités et de quelque contingent militaire, la présentation d'un kiosque décoré dans lequel figurait l'effigie d'un ou plusieurs des pères fondateurs du Mexique indépendant, la musique, le tir de salves, l'illumination nocturne, les feux d'artifice et le bal. Dans tout cela, le discours occupe une place centrale : il constitue l'affirmation du sens de toute la cérémonie. L'hommage, le culte des héros de l'Indépendance est le dénominateur commun de tous les discours; en plus, on y recherche l'unité nationale autour de ce culte, et en arrière-plan l'affirmation d'une identité fondée sur la reconnaissance des mêmes origines. Mais en même temps, les intérêts du jour s'y expriment : l'orateur représente également un groupe déterminé au sein de la nation et c'est la voix de ce groupe qui se fait entendre; il s'approprie évidemment l'héritage de la pensée et de l'oeuvre des héros, ou, pour mieux dire il adapte cet héritage à ses intérêts, généralement pour justifier le pouvoir du moment, parfois pour le critiquer. Il s'agit donc le plus souvent de la substance de la vision officielle du passé et, de temps à autre, d'une vision différente. Dans le cadre de la cérémonie, l'une et l'autre vision paraissent se confondre avec les sentiments généraux de la nation et du peuple qui réclament le culte patriotique.

4 Le schéma fondamental adopté dans ces discours comporte deux aspects : le souvenir de l'épopée et l'exhortation. Ces aspects se moulent dans les parties du discours et aux autres normes de la rhétorique en vogue. Selon celles-ci, les parties du discours qu'on avait l'habitude de distinguer étaient l'exorde, la narration, la preuve, la réfutation et la péroraison. Mais la rhétorique était encore déterminée par les courants littéraires et culturels du moment et par ceux de l'époque de la formation de l'orateur. En d'autres termes, la rhétorique aussi a son histoire. De telle sorte qu'au siècle dernier, deux tendances rhétoriques s'observent, l'une qui s'assujettit principalement aux préceptes littéraires ou à l'observation des grands modèles, et l'autre qui parait abandonner les normes et les paradigmes pour s'exprimer de manière multiforme et libre. Elles correspondent, à peu près aux périodes successives du néoclassicisme et du romantisme. Cependant, dans les discours réels, il n'est pas rare qu'elles se mêlent. Une des manifestations de la tendance à une plus grande liberté réside dans la simplification ou le développement des parties susdites : souvent, il n'y a pas de narration proprement dite, ni de preuve ou de réfutation, mais un seul élément de preuve ou bien une série d'entre eux. Les parties canoniques du discours montrent que la rhétorique ne s'épuise pas dans sa dimension littéraire; comme art de la persuasion, elle tire sa force essentielle de l'argumentation, de la logique; non seulement la logique formelle, attentive au dépouillement de la pensée correcte, mais la logique dialectique qui prend en compte l'homme tout entier, avec ses intérêts, ses sentiments et ses émotions.

5 La réitération constante du discours civique tout au long du siècle, son caractère à la fois officiel et populaire, sa fonction conformatrice de la société même qui le programme et le reçoit, son invocation particulière des origines d'une nation et d'une patrie, et enfin sa transmission comme patrimoine, inaltérable dans certains de ses aspects et sujet à des changements dans d'autres, soit pour s'adapter au rythme de l'histoire, soit pour s'enrichir ou s'appauvrir, nous conduisent à le considérer comme une tradition cardinale dans l'histoire du nationalisme. 
6 Si l'on s'en tient à la première moitié du siècle dernier, le développement de la tradition du discours civique au Mexique comprend trois périodes : les origines, de 1808 à 1825 ; la consolidation, de 1826 à 1846 ; la confrontation de la tradition à la révision de l'histoire, à l'invasion et aux guerres de la Réforme et de l'Intervention française de 1847 à 1867. Comme on peut le voir, la chronologie du discours patriotique ne coïncide pas toujours avec la chronologie politique généralement admise, bien qu'elle soit fortement influencée par elle.

7 La première période débute avec l'apparition des sermons et des discours au moment de la prestation du serment à Ferdinand VII. Bien qu'il s'agisse encore de la période coloniale, de tels textes sont un modèle immédiat pour les discours postérieurs de l'insurrection: le serment à Ferdinand VII, dans le contexte de l'occupation de l'Espagne par les troupes françaises déchaîna une vague de patriotisme enthousiaste, modelé par l'art oratoire, et prépara la diffusion du Grito de Independencia (l'appel du curé Hidalgo à l'insurrection, le 16 septembre 1810) qui en resta initialement imprégnée. La célébration rapide du Grito en pleine guerre, dès 1812, et celle de la mémoire des premiers chefs, Hidalgo et Allende, furent marquées dès l'origine par la rédaction et par la proclamation de pièces oratoires qui prirent la forme de sermons patriotiques. Nous ne sommes donc pas encore devant un discours purement civique. Le mouvement "des Trois Garanties » qui consomma l'Indépendance, ainsi que les brefs épisodes de la Régence et de l'Empire, n'échappèrent pas à ce genre mixte. D'autre part, avec le rejet du recours à la violence comme option première, on s'écarta de la célébration du Grito au profit d'autres cérémonies accompagnées de sermons patriotiques: le serment des Trois Garanties du Plan d'Iguala, l'entrée de l'Armée "des Trois Garanties » dans Mexico et le serment impérial d'Iturbide. La chute de celui-ci vit un changement important des contenus, mais non des formes: la figure d'Hidalgo et d'autres insurgés fit retour, tandis que le nom d'Iturbide était systématiquement tu. L'une des transitions entre sermon et discours s'opère lorsque Francisco Argandar prononce en 1823 un texte pour le transfert des restes des principaux pères fondateurs à la cathédrale de Mexico. Il faut attendre 1825 pour qu'apparaisse dans sa perfection le premier discours patriotique commémoratif civique.

L'influence du sermon sur le discours civique emprunte deux voies. Le sermon panégyrique, qui exalte les vertus et les prodiges de tel ou tel saint, offre un modèle mutatis mutandis pour l'apothéose des pères fondateurs. La place des démons est alors occupée par les détestables oppresseurs gachupines (ces Espagnols de la péninsule installés en Amérique) ou par le parti opposé à celui de l'orateur. Plus que d'une histoire critique, il part d'une hagiographie. On édifie l'autel de la patrie et on coule l'histoire dans le bronze. Le discours civique serait dans ce sens la version sécularisée du panégyrique religieux. Des éléments existent pour cette interprétation. L'énorme importance des sermons à l'époque coloniale et dans les débuts de l'Indépendance fit qu'inévitablement la mentalité du peuple et des orateurs eux mêmes les prenait en compte comme référence et même comme modèles obligés, non seulement pour leur orientation générale mais jusque dans des détails de ton et de tournure. Cependant, il faut préciser. S'il est certain que le sermon panégyrique était le référent le plus fréquent, les traces d'autres types de sermons se retrouvent dans quelques discours patriotiques. Je pense au sermon funèbre et au sermon d'action de grâces destinés à exalter un personnage comme le roi. Pour l'orateur civil, ces modèles sont plus accessibles que le panégyrique car ils traitent de personnages non canonisés, fréquemment remarquables non tant pour leurs vertus 
strictement religieuses que, comme les pères fondateurs, pour leurs vertus civiques ou sociales.

9 L'influence du sermon se repère encore à travers l'évolution historique qui fit du sermon patriotique un discours civique. C'est cette perspective qui m'intéresse, parce qu'elle montre de façon spécifique et vérifiable les origines d'une tradition en tant que processus temporel. Nous allons donc étudier le premier moment de la tradition du discours civique mexicain. Laissant de côté les sermons qui accompagnent le serment à Ferdinand VII, je me concentrerai sur les premières célébrations du Grito qui eurent lieu à des dates très précoces, lorsque la tête de Hidalgo était encore suspendue à la halle au blé de Guanajuato.

Le culte des héros de l'Indépendance commença avant même que celle-ci fût effective, et l'initiative formelle en revint à Ignacio Rayon. C'est lui qui, pour la première fois en 1812, organisa trois fêtes publiques patriotiques commémoratives: la première le 31 juillet, pour le jour de la fête d'Ignacio Allende ; puis le 16 septembre, deuxième anniversaire du Grito de Dolores ; et enfin le 29 septembre pour la fête de Miguel Hidalgo.

11 La première célébration eut lieu à Tlapujahua. Depuis la veille, 30 juillet, les balcons et les fenêtres étaient décorés de tentures ; il y eut une salve d'artillerie et « la nuit, on illumina toutes les rues et les deux places de la ville minière. » On installa dans un lieu spécial le portrait de Ferdinand VII, personnage fictif utile pour le succès de la cause ; à ses côtés apparaissaient deux huitains, l'un à Tlalpujahua et l'autre à Allende. Il y eut sérénade et acclamations « d'un concours nombreux de peuple... Le lendemain matin, on répéta les salves d'artillerie; les officiers et la troupe revêtirent leur uniforme de cérémonie; l'armée se forma dans le meilleur ordre; et à huit heures, S. E. Monsieur le Président / Rayon/ sortit accompagné d'un brillant cortège qui le conduisit jusqu'à l'église paroissiale, où l'on chanta la messe et le Te Deum avec la solennité correspondant au caractère auguste de la cérémonie; un pieux sermon fut prononcé par le R. P. Dr. Fr. Francisco Guerrero de l'ordre de la Merci, et quand tout fut terminé, S. E. revint à sa résidence ${ }^{8}$.»

Le sermon fut l'élément central des réjouissances du 31 juillet 1812. Comme si c'était la parole qui donnait le sens et expliquait la cérémonie. Au surplus, le contexte liturgique la sacralisait, la rendant indiscutable et vénérable. Malheureusement, le texte de ces premières réjouissances patriotico-religieuses ne nous est pas parvenu, ni non plus celui du 16 septembre de la même année, dû également au frère de la Merci Francisco Guerrero. Les éléments du programme de ce 16 septembre 1812 furent semblables à ceux de la fête d'Allende. La principale différence résida dans le lieu : Huichapan, cette fois ; la seconde fut qu'il n'y eut pas de vêpres solennelles à cause peut-être des hasards de la guerre. Pour la première fois, on commémorait le Grito. Un journaliste poète, le licenciado Andres Quintana Roo, écrivit pour l'occasion une ode. Le journal de Rayon rapporte l'événement : «Avec une salve d'artillerie et la volée de toutes les cloches, commencèrent à l'aube de ce jour les solennités pour la mémoire glorieuse du cri de liberté lancé voici deux ans dans la Congrégation de Dolores par les illustres héros et sérénissimes Hidalgo et Allende, après qu'on eut demandé la veille l'illumination et la décoration de toutes les rues. S.E. /Rayon/ assista en la brillante compagnie de son escorte, officiers et soldats, à la messe d'actions de grâce au cours de laquelle le Sr. Dr. Brigadier don Francisco Guerrero prêcha, et en même temps que la messe, l'artillerie et la compagnie de grenadiers de Huichapa tirèrent des salves. A midi, au cours de la sérénade, les deux musiques, 
rivalisant, jouèrent plusieurs morceaux choisis au goût de S.E. et à la satisfaction de tout le public9.»

\section{Saint-Michel archange et Miguel Hidalgo}

13 organisateurs et la chronique confondirent sa fête et son anniversaire, car ils
prétendaient célébrer sa naissance, qui en réalité avait eu lieu le 8 mai. Quoi qu'il en soit, on répéta mutatis mutandis les éléments du programme. L'orateur fut pourtant différent, car le prédicateur, le frère de la Merci, Guerrero, était décédé la veille. Sa place fut occupée par un ecclésiastique fougueux, qui venait de rejoindre l'insurrection au scandale du parti royaliste, car en plus d'être docteur en théologie, il était chanoine et fils d'un fonctionnaire de premier plan. Il s'appelait Francisco de Velasco et était appelé à devenir un personnage tragi-comique. Quelques jours après, le sermon fut publié à Tlalpujahua. C'est le premier texte oratoire insurgé que nous connaissions directement grâce à la réédition de Ernesto Lemoine ${ }^{10}$.

Suivant le rituel des présentations, l'éditeur-imprimeur, Josef Maria Revelo recommande un texte selon lui « riche de traits d'éloquence exquis; c'est un tissu de souvenirs très doux aux tournures majestueuses où brille une simplicité enchanteresse... et qui confond l'audace éhontée avec laquelle des détracteurs insolents prétendirent ternir la vertu du premier Père de la Patrie ${ }^{11}$ » Ceci se réfère aux accusations d'hérésie portées contre Hidalgo, en réalité jamais sanctionnées par l'Inquisition. Pour sa part, l'orateur s'en tient à l'habituelle déclaration de modestie et prétend accepter la publication " pour obéir et satisfaire d'une certaine façon à la bonté d'un auditoire » qui l'écouta avec plaisir.

L'épigraphe dont s'inspire le prêche est un verset de l'Apocalypse $(12,10)$ : «J'entendis alors une forte voix céleste dire: 'Maintenant sont arrivés le salut, la puissance et la royauté de notre Dieu, ainsi que la domination de son Christ, puisqu'on a jeté bas l'accusateur, qui accusait jour et nuit nos frères devant notre Dieu'.» Le texte était interprété comme la défaite et l'expulsion de Satan par l'archange Saint-Michel. Il n'y a pas de doute qu'il se prêtait aux circonstances. Mais laissant comme en suspens l'intéressant parallèle, l'orateur passait à un exorde d'un style cicéronien, avec une série de propositions conditionnelles qu'achève, afin de retenir la bienveillance des auditeurs, la désignation comme lieu auquel ils aspirent aussi, de « la cime de la victoire, où sont déposés l'honneur, la gloire et l'immortalité. "

Dans la proposition du discours il établit clairement le parallélisme qui forme l'épine dorsale du discours: comme l'Église célèbre le triomphe de Saint-Michel, la Patrie remémore le glorieux haut fait de l'autre Michel. Tous deux luttèrent contre l'esprit de superbe arrogant et contre l'intérêt sordide : l'archange contre la superbe luciférienne, le mexicain contre la superbe métropolitaine. Dans ces conditions, quel tribut devra être payé à la mémoire du « premier héros de la patrie »?

17 L'argumentation, conséquemment, se déroule en deux parties, la narration et la preuve, subdivisée chacune à son tour en deux. D'abord, ce qui concerne Saint-Michel: a/ l'orgueil, « génie premier né de l'abîme », pénétra « un esprit de premier ordre », qui osa se dresser contre Dieu ; b/ le ciel tressaillit. Un prince céleste cria « qui comme Dieu ? » et la lutte commença. Lucifer fut chassé. Ici est répétée l'épigraphe inspiratrice (Apocalypse, $12,10)^{12}$. 
18 La seconde partie, la preuve, relative à Miguel Hidalgo, se déroule ainsi: a/ l'esprit d'orgueil s'empara de cette terre avec l'humiliation pour résultat, si bien qu'une " obéissance sans exemple et une fidélité étonnante nous faisaient baiser la main même qui nous frappait ». Quand la perfidie arracha du trône Ferdinand, l'Amérique demeura trompée et soumise à "une dure servitude.../à une/ tyrannique oppression»; b/ Dieu suscita un génie singulier et extraordinaire, "un esprit supérieur », Hidalgo, qui dut dépasser le «mur de bronze » qui, du fait de sa position et de son âge, le séparait de la guerre. Mais » il entendait partout la voix de la patrie opprimée ». Et du fait de sa « haute réputation", les peuples le reconnurent et le proclamèrent leur libérateur ${ }^{13}$. Un autre modèle s'offre alors irrésistiblement à l'orateur : il rapproche la figure d'Hidalgo devenu chef de celle de Moïse à la tête du peuple d'Israël ; cette idée implique celle de peuple élu, qui sera reprise fréquemment par plusieurs insurgés, Morelos particulièrement. L'orateur, Velasco, poursuit en montrant comment devant le gouvernement d'Hidalgo se dressa l'infamie de ses accusateurs, mais comment ceux-ci furent incapables d'empêcher qu'à travers triomphes et déroutes son projet fut repris au-delà même de sa mort.

19 La réfutation et la péroraison, à vrai dire, ne parviennent pas au niveau du développement précédent. La réfutation se réfère seulement à des triomphes et des revers du fait des raisons impénétrables du Très Haut, tandis que la péroraison achève précipitamment avec une invocation à la Vierge de Guadalupe, la priant de protéger le mouvement, comme une "cause sienne ${ }^{14}$ ». Il existait des éléments pour une récapitulation vigoureuse et une exhortation solennelle.

20 Quoi qu'il en soit, le sermon de Velasco est de la plus grande importance, puisqu'il inaugure la tradition du culte patriotique au Mexique. Les sermons précédents du frère Guerrero furent emportés par le vent. Celui de Velasco marque effectivement le modèle de la présentation oratoire de l'épopée, soit en chaire, soit à la tribune. Le plan dans lequel on dépeint d'abord la situation antérieure au Grito, puis les hauts faits d'Hidalgo et enfin leurs conséquences, constituera un schéma de base, véritable sentier battu dès lors. Le modèle de Saint-Michel ne sera pas très sollicité, mais celui de Moïse réapparaîtra fréquemment. Le thème de l'incroyable obéissance sera également repris par un nombre non négligeable d'orateurs. A l'inverse, la proclamation simultanée et paradoxale d'hostilité envers la métropole et d'attachement à Ferdinand ne dura que jusqu'en 1813.

Ceci étant, il me semble qu'on doive corriger l'idée selon laquelle l'inventeur du panthéon et du culte patriotiques fut Carlos Maria de Bustamente. Rayon et son entourage le devancèrent, non seulement pour la célébration publique mais aussi pour ce qui est de son principe obligatoire, véritable obsession pour l'homme de Tlalpujahua, dont les Elementos Constitucionales de 1812 comprennent ce point : « 33. Le 16 septembre, jour de la proclamation de notre heureuse indépendance, le 29 septembre et le 31 juillet, anniversaires de nos généraux en chef Hidalgo et Allende, et le 12 décembre consacré à notre très aimable protectrice, Notre-Dame de Guadalupe, seront célébrés comme les plus augustes de notre Nation ${ }^{15}$.»

\section{Le discours inaugural de Chilpancingo}

L'année suivante, en septembre 1813, le pouvoir le plus important au sein de l'insurrection était passé aux mains de Morelos. Le président Rayon avait été incapable d'empêcher les divisions au sein même du conseil de gouvernement. D'autre part, 
naturellement, les triomphes de Morelos accrurent son importance; il se rassemblait autour de lui toute une cour non seulement de militaires, mais aussi d'avocats désireux de conduire le mouvement, par la bonne voie du droit, vers les idéaux des nouvelles idées politiques de liberté et de démocratie. Parmi ces avocats, certains, comme Quintana Roo, avaient collaboré avec Rayon, et d'autres avaient adhéré plus récemment, comme Bustamante, dont l'esprit bouillonnait d'initiatives et de sentiments patriotiques. C'est lui qui eut l'idée de la convocation d'un congrès qui supplanterait le conseil de gouvernement de Rayon. Morelos accepta l'idée et Bustamante s'offrit à collaborer si nécessaire pour mener à bien son initiative.

Morelos chargea alors Bustamante de la rédaction du discours d'ouverture, et lui donna des indications précises sur l'objectif, les thèmes et même le style. Il est probable qu'il y eut un brouillon avant la version que nous connaissons, que Morelos corrigea, omettant en particulier la référence à Ferdinand VII. On suppose que Morelos prononça le discours le 14 septembre 1813. Quoi qu'il en soit, le texte possède une signification importante, car il exprime l'esprit des dirigeants de l'insurrection. Il reflète dans plusieurs de ses paragraphes la pensée de Morelos tandis que dans d'autres on reconnaît la main et les préoccupations de l'avocat Bustamante.

Le discours d'ouverture du Congrès de Chilpancingo est très particulier. Il dégage tout entier une tonalité pathétique et solennelle. Il est dominé par une idée : sauver la patrie. On aurait pu s'attendre à une harangue exhortant les députés à remplir leur mission de législateurs. Mais le discours prend de l'ampleur tout au long de trois parties. La première rappelle avec élégance les principes fondamentaux du droit des gens, et les rapporte à l'injustice de la domination coloniale ${ }^{16}$. Ce dernier point lui donnerait le caractère d'une narration.

La seconde partie, plus qu'elle ne confirme la précédente, décrit de manière épique le soulèvement armé, les sacrifices qu'il a exigés, et surtout la mort de tant de héros que l'orateur interpelle pour qu'ils reçoivent ce voeu : «Mourir ou sauver la Patrie ${ }^{17}$ !». Elle se poursuit par la description des dangers que le soulèvement a courus, spécialement l'anarchie dans les rangs des insurgés énergiquement condamnée. Elle se conclut par le récit des peines de ceux qui, exemplaires, se sont engagés pour la cause : "tout leur a manqué parfois, sauf le désir de sauver la Patrie ${ }^{18}$.»

Dans la troisième partie, qui n'a rien d'une réfutation, l'orateur se tourne directement vers le congrès, le comparant à un aigle généreux dont les ailes protectrices seraient les lois, dont les serres seraient les armées et dont les yeux perspicaces seraient une sagesse profonde (cette image correspondait à l'un des drapeaux de l'armée insurgée, sur lequel apparaissait l'aigle avec la devise : "Victorieux à la fois par le regard et par les serres ». La péroraison porte les évocations à leur plus haut degré ; elles correspondent aux deux premières racines du Mexique : en premier lieu, l'orateur invite à célébrer la vengeance des chefs indigènes de l'époque de la conquête; ensuite, il loue le "Dieu de nos pères ", évidemment celui de la religion catholique, pour ses grands bienfaits, et il débouche sur l'objectif principal du discours : faire jurer aux députés de sauver la Patrie et de maintenir la religion catholique. L'ensemble se conclut par un éloge historique solennel et par un avertissement grave : "nous allons rétablir l'Empire mexicain... Craignons le jugement d'une postérité juste et inexorable ${ }^{19}$.»

Il s'agit donc d'un texte inaugural, dont le propos essentiel est d'éveiller une émotion capable de raviver la résolution de donner sa vie pour le salut de la patrie. Avec cet esprit, 
cette valeur assimilée, toute fonction, qu'elle soit législative, militaire ou administrative, serait assumée jusqu'à ses ultimes conséquences. En outre, l'importance primordiale de cette pièce oratoire tient à ce qu'elle est le premier discours proprement civique de la nation au moment critique où elle aspire à l'indépendance, et qu'un laïc participa de manière décisive à son élaboration. Au moins s'agit-il du premier qui nous soit parvenu.

Nous n'avons donc pas affaire à un sermon patriotique comme ceux qui étaient prononcés alors et qui continueraient à l'être fréquemment pendant de nombreuses années, y compris après l'indépendance. A Chilpancingo, le caractère particulier et séculier de la lutte ainsi que la responsabilité des laïcs dans l'usage public et solennel de la parole commençaient à s'assumer et à être reconnus. Évidemment, il ne s'agit pas d'abandonner les aspects religieux, mais le premier plan n'est plus occupé maintenant par ceux-ci, mais par les valeurs propres de l'émancipation politique et de sa conquête.

Il ne s'agit pas non plus d'un discours de commémoration, mais d'un discours inaugural qui ne devrait par conséquent pas entrer dans la perspective qui est la nôtre. Pourtant, s'il n'est pas directement commémoratif, l'analyse montre qu'il contient suffisamment d'éléments du discours de commémoration d'un 16 septembre : le récit de la domination coloniale, la description épique de l'insurrection, le culte de la mémoire des premiers héros et aussi le rejet de la division interne seront des lieux communs de pratiquement tous les discours septembristes du XIX ${ }^{e}$ siècle, à tel point que le texte de Chilpancingo apparaîtra comme leur modèle, avec l'avantage sans doute que la rhétorique du texte de Morelos-Bustamante laisse transparaître l'authenticité de la vie et respire la fraîcheur du moment.

Deux jours après, le 16 septembre 1813, Bustamante lui-même publia dans le journal officiel de l'insurrection un «transport d'enthousiasme patriotique » pour le troisième anniversaire du Grito de Dolores ${ }^{20}$. Il débute par une citation du poète Young et se poursuit par une série d'allégories, d'évocations, d'interpellations grandiloquentes, où l'on note la différence entre le discours supervisé par Morelos et le génie de Bustamante laissé à sa propre impulsion. Quoi qu'il en soit, ce qui est important pour nous est la continuité de la commémoration officielle du début du mouvement insurrectionnel. Cela correspondait au désir de Morelos qui, reprenant l'idée de Rayon, venait de consacrer dans les Sentimentos de la Nacion la célébration du 16 septembre ${ }^{21}$.

Pendant les années suivantes de lutte, on continua très probablement de célébrer les jours que Morelos et Rayon avaient consacrés comme fêtes nationales. Les circonstances de la guerre ne le permirent pas toujours et partout. Des indices existent de cette continuité. Le 31 juillet 1814 , jour de la fête d'Allende, une commémoration en règle eut lieu à Zacatlan, où se trouvaient à ce moment Rayon et Osorno. Et en 1817 encore, malgré le déclin de la cause insurgée, on célébra la mémoire d'Hidalgo, à ce moment comme un rite qui semblait le gage d'une renaissance de l'espoir. Dans le fort de Jaujilla, près de Zacapu dans le Michoacan, on publiait une Gaceta où parut en septembre 1817 cette consigne : «Les commandants généraux et particuliers veilleront à ce que dans toutes les paroisses de leurs départements soit célébré le 29 de ce mois avec Te Deum et messe d'action de grâces à la mémoire de $\mathrm{S}$. A Sérénissime don Miguel Hidalgo y Costilla. Les commandants pour leur part solenniseront ce jour avec tous les honneurs militaires et les subdélégués disposeront les illuminations dans toutes les rues ${ }^{22}$.» 


\section{Les sermons de l'accession à l'indépendance et de l'empire} insurrection, dans la mesure où l'on insistait tout particulièrement cette fois sur l'union des "Américains » et des "Européens ", c'est à dire des Blancs créoles et des Espagnols nés dans la péninsule ibérique. On n'oublia pas les premiers héros : peu de jours avant l'entrée de l'armée des Trois Garanties dans la ville de Mexico, une publication officieuse interpellait ainsi les pères de la Patrie : «Finalement, héros illustres qui en 1810 aviez prononcé l'indépendance de votre patrie, l'objet de votre glorieux sacrifice s'est accompli ${ }^{23}$ ». Et l'Acte d'Indépendance se réfère expressément au Grito de Dolores. Cependant, la célébration du 16 septembre ou de ses héros fut de fait hiérarchiquement placée en 1821 et 1822 au second rang, après le 27 , jour de l'accession à l'Indépendance ou bien celle du couronnement d'Iturbide.

Je n'ai pas trouvé de discours civique imprimé daté de ces années qui célèbre l'événement. Ce qu'on trouve publié, ce sont des sermons patriotiques. Quelques uns de leurs auteurs sont conscients du caractère mixte ou hybride de leurs discours, et ils s'efforcent d'en donner une explication. Ils représentent l'avant-dernière phase du passage du genre sermon au discours clairement civique. La dispersion en plusieurs lieux de l'empire de l'ensemble de ces sermons publiés (en plus de Mexico, on trouve Guadalajara, Puebla, Zacatecas, Tepic, San Miguel el Grande et Patzcuaro) retient l'attention. La plus grande partie d'entre eux traitent des Trois Garanties et tous louent la rapidité de la campagne fondée sur ces principes ainsi que son caractère très peu sanglant, plusieurs encensent les qualités d'Iturbide ou font l'apologie de la monarchie constitutionnelle comme forme la plus adéquate de gouvernement pour la nation mexicaine.

Un des derniers sermons ou discours relatifs à l'acclamation d'Iturbide comme empereur fut prononcé le 12 décembre 1822 à Patzcuaro ${ }^{24}$. Iturbide avait été couronné en juillet. Mais, en août une conspiration avait été découverte qui avait conduit à l'arrestation de plusieurs députés et en octobre le congrès avait été dissous. Le sermon ou discours prend place ainsi dans un moment de grande effervescence politique au cours duquel sont réénoncés les thèmes de la souveraineté et de la meilleure forme de gouvernement. La position décidée et argumentée qu'adopte l'orateur en faveur de l'empire et d'Iturbide favorisa la publication rapide du texte à Mexico, qui eut lieu dans les jours où le Plan de Casa Mata ${ }^{25}$ commençait d'être diffusé. Le sermon provincial publié dans la capitale, prit ainsi une dimension nationale. De plus, son auteur était un ami de l'empereur et un de ceux qui avait influé tant sur sa conversion à la cause indépendantiste que sur l'élaboration du Plan d'Iguala. Il s'appelait Manuel de la Torre Lloreda (1776-1836). Écrivain remarqué, il appartenait au groupe des clercs éclairés de Valladolid qui, depuis la période coloniale, défendait des réformes dans tous les domaines et sans doute entretenait alors des relations cordiales avec Abad y Queipo ainsi qu'avec Hidalgo. Son principal ami était le plus important orateur du diocèse du Michoacan, Manuel de la Barcena. Ce type de préoccupations l'avait conduit à se lier avec les conspirateurs de Valladolid en 1809, raison pour laquelle il fut emprisonné. Libéré, il condamna le caractère sanglant du mouvement d'Hidalgo, mais il demeura un sympathisant de la cause indépendantiste. 
L'objectif de son discours ou sermon de 1822 consiste à persuader que la monarchie constitutionnelle est la meilleure forme de gouvernement pour le Mexique, et qu'Iturbide est le plus indiqué pour ceindre la couronne. En apparence, le thème n'était pas objet de sermon, mais de discours civique; cependant l'espace et le contexte dans lesquels il fut prononcé étaient sacrés : l'église principale de Patzcuaro (l'actuelle basilique) et une messe d'action de grâces. De ce fait, De la Torre fit précéder le texte imprimé d'une note introductive, intitulée « L'auteur de ce discours aux bons esprits », dans laquelle il aborde le problème déjà rencontré par d'autres orateurs : le rapprochement d'un thème civique et des circonstances de sermon. Cet éclaircissement donne une importance particulière au texte, car il manifeste la conscience du problème posé par la confusion de genres qui impliquaient une différence non seulement de forme mais de valorisation des arguments. Quant à la forme, l'auteur fait allusion à l'absence d'épigraphe biblique et d'invocation sacrée dans l'exorde. Mais la différence de fond consistait à ne pas confondre "les matières de religion et celles de politique », et à ne pas abuser "de l'Écriture Sainte en l'apportant pour élément de preuve pour n'importe quel sujet, même s'il fallait pour cela faire violence au texte de l'Écriture. De sorte, il convenait de distinguer les sermons, qu'ils soient dogmatiques, moraux ou panégyriques, des discours simplement politiques "ou mixtes, classe parmi laquelle le présent doit être compté26». Que signifie concrètement ce caractère mixte? N'était ce pas revenir à la confusion? Le contenu du texte l'éclaire. Les arguments dont se servira De la Torre sont essentiellement d'ordre rationnel, et non pas des arguments religieux d'autorité comme dans les sermons; ils sont cependant renforcés de parallèles bibliques. Mais les sermons procédaient généralement à l'inverse : l'argument principal tenait son autorité de l'Écriture ou de la tradition, tandis que l'argument complémentaire était de caractère naturel ou rationnel.

\section{Funérailles et apothéose des insurgés}

Dès la chute de l'empereur Iturbide, le gouvernement qui lui succéda se donna pour tâche de remplacer la célébration du 27 septembre par celle du 16. Le moyen le plus opportun pour inaugurer ce changement fut la décision de rassembler dans la capitale les restes des principaux chefs de l'insurrection. C'était une occasion propice pour que soient rendus des hommages dans les divers lieux du pays où se trouvaient les dépouilles mortelles ainsi que dans ceux par où devait passer le convoi funèbre ; il apparaîtrait ainsi que la vraie reconnaissance était due davantage à ceux qui initièrent et qui soutinrent la cause qu'à celui qui réalisa l'indépendance, qui devait être quant à lui relégué dans l'oubli. Le transfert aurait pour point d'aboutissement la cathédrale de Mexico et une importante cérémonie y aurait lieu, dont le sens devait être exprimé par la bouche de l'orateur. Ce fut Francisco Argandar (1778-1830) qui, le 17 septembre 1823 prononça un Elogio finebre de los primeros heroes y victimas de la patria ${ }^{27}$ Argandar était un homme qualifié pour une telle mission, car à sa qualité de prédicateur et de théologien, il ajoutait celle de témoin et d'acteur de la geste insurrectionnelle (comme représentant de ceux qui élaborèrent la constitution d'Apatzingan). De plus, il avait été aussi député au congrès qui avait élu Iturbide empereur, non pas dans l'anonymat mais comme un de ceux qui avaient le plus ardemment appuyé cette élection par une éloquente intervention parlementaire. De sorte que l'éloge funèbre des premiers insurgés, où il leur donnait tout le mérite de l'Indépendance tout en taisant le nom d'un Iturbide réduit à l'exil, apparaîtrait comme une espèce de palinodie. 

des partisans du nouveau régime dans un morceau d'éloquence qui ne trahirait pas l'éminence de son sujet. Dès lors, l'orateur était confronté à la définition du genre du texte. Du fait de l'espace sacré et du contexte liturgique, il s'agissait d'un sermon funèbre. Mais d'un autre côté, en raison de son thème et de ses objectifs, le discours devrait être patriotique et civil. Comme sermon funèbre, il faudrait y inclure de quelque manière la préoccupation et la prière pour le repos éternel des morts ; comme discours patriotique, il devrait insister sur l'exaltation de leurs vertus civiques exemplaires. Nous retrouvons donc le genre mixte de l'époque de l'empire. à l'un des modèles que Morelos lui-même et d'autres insurgés avaient invoqués pour justifier la cause: la guerre des Maccabées. Le ton dominant du texte que prononça Argandar fut celui du sermon sui generis. Sa structure s'articule fréquemment autour du parallélisme entre les insurgés et les Maccabées. Ce système de référence alterne cependant avec des allusions à des personnages et des événements du monde classique gréco-romain. Les périodes s'organisent en beaucoup d'endroits en phrases courtes qui rendent le discours incisif.

L'épigraphe du sermon en contient le propos général : „Célébrons avec de justes éloges la mémoire des héros qui furent si glorieux et en même temps nos pères. » (Ecclésiaste, 44,1). Ces hommes sont avant tout ceux dont les cendres se trouvaient là, face à l'auditoire. Le court exorde comporte deux parties: l'une surtout évocatoire et l'autre explicative. La première s'ouvre par une apostrophe à la mort terrible, suivie d'une énumération dramatique d'écrivains et de penseurs qui ont consacré des pages au malheur et à la mort. Une séquence rythmée et solennelle, évocatrice et présente, comme le glas des obsèques, convoque Jérémie, Milton, Young, Chateaubriand, Regnault Warin, Hervey et Cadahalzo. Cet ensemble pathétique n'est pas tant destiné à montrer l'érudition de l'orateur qu'à donner au texte un ton franchement romantique; la progression s'arrête à l'orateur lui-même : "Soi-même pour sujet ${ }^{28}$ ". Comme un bon romantique, Argandar se plaît dans les lamentations, se plaignant de ce que son destin soit de rappeler les douleurs indicibles (influence virgilienne), déclarant d'autre part très légitime ces pleurs et exprimant sa reconnaissance envers la religion qui aide à endurer le malheur. Enfin, il confère un sens moral aux éloges bibliques des grands personnages : morts, ils font porter la réprobation sur la conduite de leurs opposants et sur celle des indifférents.

La partie explicative de l'exorde justifie que les pères de la patrie auxquels hommage est rendu ont été judicieusement choisis : ce sont les principaux et les plus représentatifs. Pour l'établir, il s'appuie sur une situation semblable dans le monde classique. De façon brève et élogieuse, il décrit le catafalque et énumère les héros élus : Hidalgo, Allende, Morelos, Aldama, Abasolo, Jimenez, Matamoros, Mina, Miguel et Leonardo Bravo, Galeana, Moreno et Rosales. Un doute assaille le prêtre orateur : que pensera-t-on de cet hommage rendu dans une enceinte sacrée et selon un rite destiné plutôt à intercéder pour le pardon des fautes? Il répond sans hésitation: leur sacrifice le légitime. Louons, donc, les hommes glorieux ${ }^{29}$.

41 Le premier argument se développe en deux parties, l'une générale et l'autre spécifique. Dans la première, Argandar rappelle la dévastation de la patrie et les vexations causées par le pouvoir espagnol. Mais il n'abonde pas dans ce sens et ne se complaît pas à raviver les plaies, chose très fréquente par contre chez les orateurs des années suivantes. Argandar impute même la principale responsabilité à d'autres : « trône que des ministres

Les Cahiers du Centre de Recherches Historiques, 14-15 | 1995 
sans pitié rendirent si odieux ${ }^{30} »$. Il s'étend surtout sur les triomphes et les défaites de la guerre biblique des Maccabées qui agit comme parabole du Mexique insurgé. Les héros correspondants donnent l'occasion d'une louange éloquente des "générations prodigieuses » qui se donnèrent rendez-vous dans la geste des insurgés, comme si à un moment donné les constellations célestes s'étaient rapprochées ${ }^{31}$.

Il poursuit par l'analyse et la louange particulière de chacun des pères fondateurs. A propos d'Hidalgo, il rapporte des qualités de ses jeunes années ignorées de ses biographes : « dès ses premières années, il était imperturbable face aux dangers, sagace, robuste et noblement audacieux. Quand il faisait usage de sa lance, personne ne le dépassait dans les incursions, parmi les brutes sanguinaires qu'il renversait, se moquant de leurs assauts et de leur fureur ». Il rend compte ensuite de ses études et de son magistère, de son ministère et des fabriques qu'il implanta. Le témoignage d'Argandar est confirmé : «La crainte de me faire suspecter me dissuaderait si moi, disciple parmi les disciples de cet homme insigne, et dans le palais même de Minerve, je n'avais entendu de mes maîtres et d'autres hommes impartiaux et savants des éloges encore supérieurs... ${ }^{32}$ » Pour finir, l'orateur inscrit la décision d'Hidalgo de prendre la tête du mouvement dans une réflexion supposée de ce dernier sur des passages bibliques, tous néanmoins tirés de l'Ancien Testament. Il fait rapidement le récit de l'itinéraire du Père de la Patrie associé à Allende, Aldama, Abasolo et Jimenez. Ici et là, il établit des références aux Maccabées. Il attire l'attention sur l'attitude d'Hidalgo dirigeant l'assaut de Granaditas et pleurant ensuite sur les excès qui suivirent sa conquête; de la même manière, il souligne sa sagesse, motif d'admiration à Guadalajara, tandis que son avis est rejeté avant la bataille de Caldéron.

43 Morelos mérite aussi un éloge particulier. Ses compagnons, Matamoros, Galeana et les Bravo, offrent l'occasion de poursuivre le parallélisme avec les Maccabées. Il détache, comme l'exploit le plus mémorable, la rupture du siège de Cuautla, dont il donne une description dantesque. Le rôle politique de Morelos apparaît comme un antidote à la division des insurgés : "réconcilier ses frères et les soumettre à une nouvelle forme populaire de gouvernement ${ }^{33}$ ». Il ne cite pas de noms, mais nombreux sont ceux qui savaient qu'il s'agissait de Rayon et de Berdusco. Mina, associé à Moreno, reçoit des éloges enflammés, dans un parallélisme continué avec les Maccabées. Il faut remarquer la religiosité du courageux et libéral Mina, qui « avait jusqu'à des scrupules à entendre une musique profane, ne participait pas à des divertissements les vendredis, du fait du souvenir qu'il vénérait de la Passion du Sauveur ${ }^{34}$.»

Le second argument est plus bref et se réfère aux désastres et aux défaites, à la mort des insurgés, ou mieux à leurs vertus et à leur grandeur dans la tragédie. Il commence par une série d'images de la nature : « le printemps est passé... Le ciel est devenu de bronze... De gros nuages inféconds nous recouvrent...; l'astre du jour, auparavant si bénéfique, flétrit nos $\operatorname{lis}^{35}$ ». Il se poursuit par des allusions aux héros du monde classique, et débouche enfin sur l'arrestation et le supplice d'Hidalgo et de ses compagnons, qui indignent à tel point l'orateur qu'il lance des malédictions sur Coahuila et Chihuahua. L'exécution d'Hidalgo est décrite pas à pas et on souligne les particularités de l'emprisonnement de Abasolo. A nouveau, Morelos retient particulièrement l'attention. L'orateur le suit à partir de la défaite de Valladolid et souligne ses efforts pour restaurer les forces des insurgés. Son procès et sa mort sont aussi l'objet de commentaires détaillés. Enfin Mina et Rosales apparaissent dans leur fin héroïque. Celle du second manifeste en particulier des ressemblances remarquables avec des épisodes des Maccabées. 

prières demandent le repos éternel.

C'est sous la forme de réflexion générale que l'on retrouve la préoccupation énoncée dans l'exorde: les héros immolés ont-ils été accueillis par Dieu ?.Argandar récapitule leurs vertus et conclut comme dans une ritournelle par l'épigraphe: " Faisons l'éloge des hommes illustres ». Il la confirme en rappelant le principe évangélique selon lequel donner sa vie pour les siens est la plus grande des perfections.

Dans la péroraison, un autre lien est établi avec l'exorde : que les citoyens qui l'écoutent laissent couler leurs larmes devant la douleur, énorme et légitime. Ainsi attendris, ils sont conduits par Argandar à repousser la haine envers les Espagnols, faisant valoir que les ossements présents interdisent de haïr: « Prends garde d'injurier ceux qui nous persécutèrent ${ }^{36}$ \%. Il poursuit avec une invite particulière à chacun de ceux qui se trouvent là ; pour chacun, les héros ont un message : jeunes, sages, valeureux, prêtres, philosophes, Espagnols, créoles. Les avertissements les plus solennels sont pour ceux-ci. Il réitère la nécessité de l'union et ajoute l'exigence de justice et de miséricorde, valeurs en résonance avec la pensée de Morelos : «Si confiants dans votre fortune, vous opprimiez vos semblables ou si vous leur imposiez de passer leurs jours dans l'ignominie, le Dieu de la guerre dira: Moi, alors je susciterai les plus grands ennemis qui, sans compassion, feront couler votre sang, réduiront vos fils en esclavage et votre sol en cendres ${ }^{37}$ ».

«Intercédez pour vos héritiers » est la supplique finale adressée à ceux pour lesquels les

\section{Le discours patriotique sort sur la place publique}

8 Le 16 septembre 1825 , le discours patriotique sort enfin sur la place publique pour y rester définitivement. C'est le premier anniversaire du Grito célébré par la République déjà établie, et organisé de manière formelle par le gouvernement lui-même en un acte civique. Il est vrai cependant que pendant plusieurs décennies des sermons patriotiques continueront d'être prononcés, mais ils seront de moins en moins nombreux par rapport aux discours commémoratifs prononcés par des laïcs dans un contexte civique séculier.

Un intellectuel et journaliste se mêlant parfois de politique, l'avocat Juan Wenceslao Barquera (1779-1840) inaugura cette première étape des discours civiques mexicains ${ }^{38}$. Ils appartenaient toujours au sous-genre démonstratif ou épidictique. Barquera a pour but de persuader son auditoire d'accepter qu'Hidalgo et le 16 septembre sont les symboles de l'indépendance. Il cherche implicitement à renvoyer Iturbide dans l'oubli, et à placer dans une position seconde le 27 septembre. Même si les figures du libérateur et de son entrée dans Mexico étaient encore proches. A vrai dire, on avait beau vouloir exalter la figure d'Hidalgo, celui-ci restait un inconnu pour le peuple de Mexico; par contre Iturbide avait été acclamé à plusieurs reprises par les foules citadines. Cette fois, pour éliminer le fusillé de Padilla, on se servirait de l'arme du silence. Ce qui appuyait un jugement historique : non seulement on condamnait Iturbide en le dépouillant de son existence historique même, mais encore on justifiait le gouvernement qui l'avait sacrifié, fondamentalement le même qui conservait le pouvoir avec à sa tête Victoria, qui avait l'allié décisif de Santa Anna dans le renversement d'Iturbide. L'exaltation d'Hidalgo, prédécesseur de Morelos et de Victoria, successeur de ce dernier, renforçait la consécration et l'utilisation d'une histoire destinée à légitimer le gouvernement en place.

Cependant, l'orateur ne va pas encourager à imiter l'exemple des héros (il y aurait le risque d'innombrables insurrections) mais à poursuivre le développement socio- 
politique, à lui donner un autre rythme, réellement différent, à aller toujours plus loin grâce à l'exercice de vertus affirmées. En ce sens, c'est en partie seulement que l'on prend en charge le soin de défendre ce qui a déjà été gagné. Bien que les vertus des héros soient décrites les unes après les autres, il ne s'agit pas d'inciter principalement à les imiter. On cherche surtout à persuader les auditeurs de pratiquer l'union, la vertu, le respect des lois, et particulièrement celui de la religion nationale, qui coïncide avec la religion évangélique, naturelle et universelle; et aussi à pratiquer la vertu du travail et la promotion des classes nécessiteuses; et enfin le respect de l'armée qui doit garantir le développement souhaité.

Dans l'ensemble, ce discours de Barquera représente de manière adéquate par sa forme le goût épuré d'un spécialiste expérimenté de la parole : pureté du langage et rythme des mots et des parties du discours, perspicacité dans le développement des idées et élégance cultivée, accompagnées d'une vigueur et d'un éclat classiques. Au total, il me semble que l'exorde est mieux réussi que la péroraison qui, si elle n'est pas méprisable, apparaît un peu décevante : face au ton sublime de plusieurs passages du discours, la fin reste dans le mode mineur.

Les idées exprimées dans l'ensemble du discours reflètent le libéralisme cultivé de l'auteur ainsi que ses préoccupations politiques concrètes, reflet aussi des jugements de la Junta Civica, qui semble-t-il prépara soigneusement la cérémonie commémorative (ainsi des silences sur Rayon et Iturbide, qui laissent apparaître une consigne partisane). Il faut remarquer la déclaration précoce en faveur de la tolérance ; bien qu'il vante le respect de la religion nationale, c'est à dire: la religion catholique, celle-ci n'est autre, dans le sentiment de Barquera, que la religion naturelle, en fin de compte la religion universelle, «consolation et vie des esprits rationnels»-expressions qui chez Barquera appartiennent à la maçonnerie, dans la loge écossaise de laquelle il était initié. Christianisme et maçonnerie se conjuguent: «Aimons tous les hommes, quelles que soient leur origine et leur croyance ${ }^{39}$ ». L'intérêt de Barquera pour l'amélioration du sort des classes nécessiteuses et pour la prise en compte de la participation des femmes dans "les chemins de la prospérité nationale " retiennent de la même manière l'attention ${ }^{40}$. Bien qu'il s'agisse du plus ancien discours civique, c'est celui qui se réfère dans les termes les plus positifs à la femme. La majorité des orateurs septembristes ne lui prêteront pas d'attention. Le libéralisme de Barquera inclut des préoccupations sociales. L'insistance avec laquelle il se réfère à l'armée, à laquelle il réserve la fin significative du discours, indique avec clairvoyance et non sans préoccupation le terrible pouvoir que les forces armées exerceraient dans le pays naissant.

(Traduction Jacqueline Maltrou)

Le professeur Carlos Herrejon Peredo enseigne au Collège du Michoacan, à Zamora au Mexique, où il s'occupe plus particulièrement de l'histoire de l'Église ainsi que de celle de la pensée politique mexicaine.

Il est l'auteur de nombreux ouvrages, parmi lesquels on signalera :

Textos Politicos en la Nueva Espana, Mexico, 1984.

Los Origenes de Guavangareo Valladolid, Zamora, 1991.

Hidalgo. Razones de la insurgencia y biografia documental, Mexico, 1987.

Morelos, Zamora, 1984-1987, 3 vol.

Il a séjourné au CRH comme Directeur d'études associé en mai 1995. 


\section{NOTES}

1. Charles Perelman et L. Olbrechts-Tytecal, Traité de l'Argumentation. La Nouvelle Rhétorique, Bruxelles, $5^{\mathrm{e}}$ ed., 1989.

2. H. Lausberg, Elemente der Literarischen Rhetorik, Munich, 1963.

3. R. H. Roberts et J. M. M. Good, The Recovery of Rhetoric Persuasive Discourse and Disciplinary in the Human Sciences, Londres, 1993.

4. J. Saugnieux, Les Jansénistes et le renouveau de la prédication dans l'Espagne de la seconde moitié du XVIII ${ }^{\mathrm{e}}$ siècle, Lyon, 1976.

5. M. Fumaroli, L'âge de l'éloquence, Rhétorique et « res literia " de la renaissance au seuil de l'époque classique, Paris, 1994.

6. La Conciencia National y su Formacion. Discursos civicos septembrinos (1825-1871). Recueil et avantpropos de E. de la Torre Villar avec la collaboration de R. de Anda. Mexico, 1988. E. Plasencia de la Parra, Independecia y nacionalismo a la luz del discurso conmemorativo (1825-1867), Mexico, 1991.

7. L. Moreno Valle, Catalogo de la Coleccion Lafragua de la Biblioteca Nacional de Mexico 1821-1853, Mexico, 1975.

8. Illustrador Americano, Tlalpujahua, $\mathrm{n}^{\circ} 20,1^{\mathrm{er}}$ Août 1812, p. 57-60, dans G. Garcia, Documentos Historicos Mexicanos, Mexico, III, 1910.

9. Diario dans La independencia segun Ignacio Rayon, Mexico, 1985, p. 69.

10. F. L. de Velasco, Sermon que en el cumpleanos del serenisirno senor don Miguel Hidalgo y Costilla heroe de la patria, dijo..., Tlalpujahua, 1812, dans E. Lemoine, "Sobre los fondos del AGNM referentes a la Revolucion de 1810. Documentos sobre la Revolucion de 1810 », Boletin del Archivo General de la Nacion, Mexico, IV, juillet-septembre 1980, p. 22-27.

11. Ibid., p. 22.

12. Ibid., p. 23.

13. Ibid., p. 24.

14. Ibid., p. 25.

15. E. Lemoine Villicana, Morelos. Su vida revolucionaria a traves de sus escritos y de otros testirnonios de la epoca, Mexico, 1985, p. 225.

16. Ibid., p. 355-366.

17. Ibid., p. 367.

18. Ibid., p. 368.

19. Ibid., p. 369.

20. Correo Americana del Sur, Oaxaca 16 septembre 1813, n 30, p. 233, dans Garcia, Documentos, op. cit., IV.

21. Lemoine, Morelos, op. cit., p. 372-373.

22. Gaceta Extraordinaria del Gobierno Mexicano en las Provincias del Poniente, 16 septembre 1817, dans Garcia, Documentos, op. cit., IV.

23. Diario Politico Militar Mexicano, San Bartolomé Naucalpan, 17 septembre 1821, I, nº 17, p. 73, dans Garcia, Documentos, op. cit., IV.

24. M. de la Torre Lloreda, Discurso que en la Misa de Gracias celebrada en la Iglesia Mayor de la ciudad de Patzcuaro el dia 12 de diciembre de 1822, a consecuencia de la aclamacion religiosa del Senor don Agustin Primero Emperador de Mexiro dijo..., Mexico, 1823.

25. Plan de Casa Mata: programme politique du soulèvement qui provoqua l'abdication d'Iturbide.

26. Ibid., p. 5 
27. F. Argandar, Elogio Funebre de los Primeros Heroes y Victimas de la Patria que el 17 de septiembre de 1823 en la Iglesia Metropolitana de Mexico a presencia de una Diputacion del Soberano Congreso, del Supremo Poder Ejecutivo, dernas Corporaciones y Oficialidad dijo el Dr. Francisco Argandar, diputado por Michoacan, Mexico, 1823.

28. Ibid., p. 2.

29. Ibid., p. 9.

30. Ibid., p. 10.

31. Ibid., p. 14.

32. Ibid., p. 15-16.

33. Ibid., p. 30.

34. Ibid., p. 33.

35. Ibid., p. 35.

36. Ibid., p. 48.

37. Ibid., p. 49.

38. J. W. Barquera, Oracion Patriotica, Mexico, 1825, dans La Conciencia Nacional, op. cit., p. 21-29.

39. Ibid., p. 27.

40. Ibid., p. 28. 